\title{
REVIEW
}

\section{Bosutinib for Chronic Myeloid Leukemia}

Massimo Breccia · Gianni Binotto

To view enhanced content go to www.rarecancers-open.com

Received: July 18, 2015 / Published online: August 26, 2015

(C) The Author(s) 2015. This article is published with open access at Springerlink.com

\section{ABSTRACT}

In recent years the availability of several tyrosine kinase inhibitors (TKI) in the therapeutic armamentarium for chronic myeloid leukemia has dramatically changed the objectives and expectations of healthcare providers and patients. For many, but not all, patients the forerunner of TKI, imatinib, is still an excellent treatment option. Unfortunately, nearly $30-40 \%$ of imatinib-treated patients discontinue therapy in the long-term, because of failure and/or intolerance. Second-generation tyrosine kinase inhibitors are more potent drugs which are suitable for treatment of approximately $50 \%$ of patents for whom imatinib is unsuitable, and with high success and rapid responses. Bosutinib, an orally bioavailable Src/Abl tyrosine kinase inhibitor, has proved to be effective in vitro against

M. Breccia $(\square)$

Department of Cellular Biotechnologies and

Hematology, Sapienza University, Via Benevento 6, 00161 Rome, Italy

e-mail: breccia@bce.uniroma1.it

G. Binotto

Department of Medicine, Hematology and Clinical Immunology, Padua School of Medicine, Padua, Italy resistant chronic myeloid leukemia cells that do not harbor the T315I or V299L ABL kinase domain mutations. During clinical development the manageable safety profile of bosutinib have become evident for both simple and more advanced treatment. In this review we summarize preclinical and clinical data for bosutinib and discuss its ideal field of action in comparison with other TKI.

Keywords: Bosutinib; Chronic myeloid leukemia; Efficacy; Imatinib; Safety

\section{INTRODUCTION}

The Src and Abl families of non-receptor protein tyrosine kinases have been extensively studied as targets for anticancer therapy because of their involvement in signaling pathways promoting tumor growth and progression [1-3]. Moreover, the presence of the constitutively active chimeric protein $\mathrm{BCR}-\mathrm{ABL}$, an oncogenic product arising from reciprocal translocation between chromosomes 9 and 22 (Philadelphia chromosome) [4], is regarded the pathogenetic characteristic of chronic myeloid leukemia (CML). 
Selective inhibition of BCR-ABL autophosphorylation and phosphorylation of its substrates by tyrosine kinase inhibitors (TKI) resulted in a substantial and dramatic improvement of survival of CML patients, and has become one of the most important examples of target therapy [5].

Nowadays, paradoxically (although luckily) treatment of CML has become more complicated, because of the availability of second and third-generation TKI, which are used as both salvage therapy and alternative simple options [6].

Several studies have confirmed the long-term efficacy and manageable safety profile of imatinib, the first approved TKI. However, approximately $40 \%$ of patients have to switch to different treatment because of intolerance or resistance $[7,8]$. Second-generation TKI have resulted in a favorable outcome for approximately half of non-responding patients after primary or secondary resistance $[9,10]$. In addition, use of new TKI as initial treatment resulted in improved efficacy with evidence of an extremely good molecular response and lower progression, irrespective of features and risk at diagnosis, but with inconsistent long-term overall survival compared with imatinib [11, 12].

Bosutinib, a second-generation dual inhibitor of Src and Abelson (Src/Abl) kinases, is currently approved in Europe and USA for treatment of adult patients with chronic phase (CP), accelerated phase (AP), and blast phase (BP) Philadelphia chromosome-positive CML previously treated with one or more TKI and for whom imatinib, nilotinib, and dasatinib are not regarded as appropriate treatment options. The purpose of this review is to focus on efficacy and safety data for bosutinib for treatment of resistant and/or intolerant CML or for newly diagnosed CP-CML patients.
This article is based on previously conducted studies and does not include any new studies of human or animal subjects performed by any of the authors.

\section{MECHANISM OF ACTION}

Bosutinib is an orally bioavailable $4-[(2,4-$ dichloro-5-methoxyphenyl)amino]-6-methoxy-7[3-(4-methylpiperazin-1-yl)propoxy]quinoline-3carbonitrile originally identified by Boschelli, in 2001, to act as a Src tyrosine kinase inhibitor [13]. Two years later, Golas documented potent antiproliferative and pro-apoptotic activity of bosutinib, at concentrations between 1 and $20 \mathrm{nM}$, against CML culture (K562, KU812, and Meg-01) [14]. Reduced BCR-ABL, CrkL, STAT5, and Lyn phosphorylation were consistent with both Src and Abl kinase inhibitory activity. Bosutinib was demonstrated to bind the kinase domain of BCR-ABL in an active or inactive conformation. Puttini and colleagues reported the activity of Bosutinib against BaF3 murine myeloid cells expressing resistant forms of BCR-ABL, with mutations Y253F, E255K, and D276G; no inhibition was observed against cells expressing the T315I or V299L mutation [15]. Later studies by Konig et al. focused on bosutinib activity against specific CML progenitors, and reported effective BCR-ABL and Src kinase inhibition in CML progenitor cells and growth suppression of CML primitive and committed progenitor cells. However, bosutinib did not significantly inhibit non-dividing CML primitive progenitors [16]. In 2009, by use of a chemical proteomics approach in combination with in-vitro kinase assays against a large number of recombinant kinases, Remsing Rix provided more insight into bosutinib's kinase target profile. Bosutinib, similar to dasatinib, was shown to target TEC 
family kinases, including BTK; however, in contrast with dasatinib, bosutinib did not inhibit KIT or PDGFR, but rather had activity against the STE family of kinases, in particular the STE20 subfamily. CAMK2G, a $\mathrm{Ca}^{2+} /$ calmodulin-dependent protein kinase was also identified as a novel kinase target inhibited by the drug [17].

\section{PHARMACOKINETIC DATA}

The pharmacokinetic (PK) profile of the drug in the orally bioavailable form was extensively studied in phase I/II trials among either adult healthy volunteers of cancer patients. Population PK analysis of three clinical studies among patients with cancer suggested that baseline characteristics (age, body weight, gender and race) did not affect the PK data. Drug absorption was relatively slow, with a median time to peak concentration of $4-6 \mathrm{~h}$ and a half life $t_{1 / 2}$ ranging from 33 to $39 \mathrm{~h}$, thus supporting a once-daily dosing regimen [18].

The effect of food was studied among 55 healthy subjects randomly assigned to receive bosutinib 200, 400, 600, or $800 \mathrm{mg}$ with food or 200 or $400 \mathrm{mg}$ without food, or placebo [19]. Significant increase in bosutinib exposure was observed for maximum serum concentration $\left(C_{\max }\right)$, and area under the curve (AUC) increased by $1.6-1.7$-fold when taken with food, the effect being more evident at lower doses. The interaction, resulting in increased drug exposure, was explained by an increase in bosutinib solubility when taken with food [19].

After administration of a single dose of $500 \mathrm{mg}$ bosutinib, mean apparent volume of distribution for patients with CML was $6080 \pm 1230 \mathrm{~L}$, which correlated with extensive partitioning into tissues. Bosutinib is highly bound to human plasma proteins in vitro (94\%) and ex vivo in healthy subjects
(96\%), and binding was not concentrationdependent. The major circulating metabolites identified in plasma were oxydechlorinated (M2) bosutinib (19\% of parent exposure) and $\mathrm{N}$-desmethylated (M5) bosutinib (25\% of parent exposure), with bosutinib $\mathrm{N}$-oxide (M6) a minor circulating metabolite. All the metabolites were inactive [20].

For patients with CML given single oral doses of $500 \mathrm{mg}$ bosutinib with food, the mean terminal phase elimination half-life was $22.5 \mathrm{~h} ; 91.3 \%$ of the dose was recovered in the feces and $3 \%$ in the urine. Undergoing extensive first-pass-metabolism, bosutinib was deemed to interact with CYP3A inducers and inhibitors. Early studies showed that bosutinib is primarily metabolized by hepatic CYP3A4. In a trial of 24 healthy volunteers, a single dose of $100 \mathrm{mg}$ bosutinib was administered either alone or in combination with 5 daily doses of $400 \mathrm{mg}$ ketoconazole under fasting conditions. Ketoconazole increased bosutinib $C_{\max }$ and AUC 5.2-fold and 8.6-fold, respectively. Moreover, co-administration reduced the mean apparent clearance of bosutinib approximately ninefold and increased the mean terminal half-life from 46.2 to $69.0 \mathrm{~h}$. Despite this increase in bosutinib exposure, the incidence of adverse side effects was comparable with that for administration of bosutinib alone [21]. In a cross-over trial of 24 healthy volunteers, a single dose of $500 \mathrm{mg}$ bosutinib was administered alone or in combination with six daily doses of $600 \mathrm{mg}$ of rifampicine, a potent CYP3A4 inducer, under fed conditions. Rifampicine decreased bosutinib $C_{\max }$ and AUC by $86 \%$ and $94 \%$, respectively [22]. According to these data, concurrent use of bosutinib with strong or moderate CYP3A inhibitors (and inducers) should be avoided whenever possible. Furthermore, P-glycoprotein (P-gp) inhibitors and grapefruit or grapefruit juice 
should be avoided because their administration may result in increased drug plasma concentrations. In-vitro data showed no effect of bosutinib as an inducer or inhibitor of the metabolic liver enzymes CYP1A2, CYP2A6, CYP2C8, CYP2C9, CYP2C19, CYP2D6, or CYP3A4.

Interaction of bosutinib with gastroprotective drugs was tested in a trial with 24 healthy fasting subjects. A single 400-mg dose of bosutinib was given concurrently with repeated doses of lansoprazole $60 \mathrm{mg}$. Bosutinib $C_{\max }$ and AUC decreased by $46 \%$ and $26 \%$, respectively [23]. Therefore, concomitant administration of proton-pump inhibitors with bosutinib should be avoided whenever possible; otherwise, short-acting antiacids or histamine-2 receptor antagonists should be considered if taken $2 \mathrm{~h}$ before or after bosutinib.

The relationship between bosutinib exposure at steady state and most common adverse side effects (i.e., diarrhea, thrombocytopenia, rash, transaminases (ALT/AST) increase, nausea, vomiting, and neutropenia) was recently investigated by Hsyu et al., who combined data from phase III and phase I/II clinical studies on 749 patients with newly diagnosed CP-CML or with CP-CML resistant and/or intolerant to previous imatinib therapy, respectively [24]. Associations between bosutinib exposure at steady state and key efficacy endpoints from each of the two studies were also investigated. An exposure-response relationship was identified for the incidence (but not severity) of diarrhea; a weak relationship was also observed for the incidence of rash. No evidence of an exposure-response relationship was documented for nausea, vomiting, neutropenia, thrombocytopenia, or liver enzymes elevation. For patients with newly diagnosed CP-CML, exposure-response relationships were observed for complete cytogenetic response at 1 year (predicted probability, 0.476-0.650), major molecular response (MMR) at 1 year (0.238-0.497), and cumulative complete hematologic response (CHR) at 1 year (0.605-0.763). For patients with previously treated CP-CML, no exposure-response relationship was observed for major cytogenetic response (MCyR) at 24 weeks (0.320) [24].

Pharmacokinetic of bosutinib in the context of hepatic impairment was investigated in a dedicated trial in which a single dose of $200 \mathrm{mg}$ was administered with food to 18 volunteers with A, B, and C Child-Pugh classes and to 9 matched healthy volunteers. $C_{\max }$ of bosutinib was found to be increased 2.4-fold, twofold, and 1.5-fold, respectively, for Child-Pugh classes A, B, and C, and bosutinib AUC increased 2.3-fold, twofold, and 1.9-fold, respectively. On the basis of these data, use of bosutinib is contraindicated in Europe for patients with hepatic impairment [25].

The effect of renal impairment on bosutinib pharmacokinetic profile was evaluated in a phase-1 two-stage trial in which a single dose of $200 \mathrm{mg}$ was administered, with food, to 26 subjects with mild (CLcr $51-80 \mathrm{~mL} / \mathrm{min}$ ), moderate (CLcr $30-50 \mathrm{~mL} / \mathrm{min}$ ), or severe (CLcr $<30 \mathrm{~mL} / \mathrm{min}$ ) renal impairment and to 8 subjects with normal renal function. Although bosutinib exposure was unchanged for subjects with mild renal impairment, moderate and severe renal impairment were associated with increases in AUC of $35 \%$ and $60 \%$, respectively, compared with subjects with normal renal function. Specific recommendations concerning dose adjustment were made for patients with severe (CLcr $<30 \mathrm{~mL} / \mathrm{min}$ ) or moderate (CLcr between 30 and $50 \mathrm{~mL} / \mathrm{min}$ ) renal impairment [26]. 


\section{PHASE 1/2 STUDY: CLINICAL EFFICACY}

In the phase 1 portion of a phase $1 / 2$ study that enrolled imatinib-treated CML patients, the bosutinib dose of $600 \mathrm{mg} / \mathrm{d}$ was the maximum tolerated dose. Part 1 was a dose-escalation study with $3+3$ design with subsequent cohorts of 3-6 imatinib-resistant patients. The dose of $500 \mathrm{mg}$ once-daily was selected for phase 2 of the trial; the trial was later amended to include nilotinib and dasatinib-treated patients when these drugs became commercially available. Escalation to $600 \mathrm{mg}$ daily was allowed after lack of efficacy (failure to achieve CHR by week 8 or complete cytogenetic response (CCyR) by week 12). Imatinib resistance was defined as no hematological improvement within 4 weeks, no CHR by 3 months, no cytogenetic response by 6 months, or no MCyR by 12 months, for at least $600 \mathrm{mg}$ imatinib daily. Imatinib intolerance was defined as grade IV hematologic toxicity lasting for more than 7 days, grade 3-4 non-hematological toxicity, or grade 2 toxicity that did not improve despite adequate management or adjustment of the dose of the drug. The primary endpoint of the study was MCyR at 24 weeks for patients with no previous TKI exposure other than imatinib.

Part 1 included 17 patients with imatinib resistance in the chronic phase and 1 accelerated-phase patient: bosutinib was well tolerated without dose limiting toxicity (DLT) in the 400-500 mg cohorts; indeed, in the $600 \mathrm{mg}$ cohort, one patient developed vomiting, rash, and nausea related to the drug. In the second part of the trial, the median dose intensity reported was $484.9 \mathrm{mg} /$ day for imatinib-resistant and $394.1 \mathrm{mg} /$ day for imatinib-intolerant patients.

Overall, for the 200 imatinib-resistant and 88 imatinib-intolerant CP-CML patients enrolled in this trial, after 1 year, CHR was achieved for $86 \%$ of imatinib-resistant and $85 \%$ of imatinib-intolerant patients; this was sustained for $72 \%$ and $87 \%$, respectively and was obtained in a median time of 2 weeks. Fifty-four percent of imatinib-resistant and $49 \%$ of imatinib-intolerant patients had a decrease in $\mathrm{Ph}+$ metaphases to $<35 \%$ (MCyR); this was maintained for $72 \%$ of imatinib-resistant and $92 \%$ of imatinib-intolerant patients. Median time of MCyR was 12.3 weeks. CCyR was detected in 41\%; among those patients who achieved CCyR and were evaluable for molecular response, $64 \%$ of imatinib-resistant and $65 \%$ of imatinib-intolerant patients achieved a MMR. Complete molecular response (CMR) was achieved by $49 \%$ and $61 \%$ of patients, respectively [27]. Mutational analysis was assessed for 115 patients at baseline (most frequently observed M351T, F359V, F317L, L248V, G250E, M244V, T315I) and responses were observed for all mutants except T315I [27]. At 1 year, progression-free survival (PFS) was 91\% and overall survival (OS) was $97 \%$ [27]. After 2 year, CCyR was $48 \%$ and MMR was 35\%, not expressed on international scale (IS), with $28 \%$ of patients achieving CMR. Median time reported to achieve MMR was 35.9 weeks for imatinib-resistant and 12.2 weeks for imatinib-intolerant patients. At 2 years, PFS was 81\%; progression to blast phase occurred for 11 patients and 2-year OS was 91\% [28] (Table 1).

Long-term outcome for patients treated in the advanced phase of disease has recently been reported for 79 patients in the accelerated phase (AP), 64 in the blast phase (BP), and 24 with acute lymphoblastic leukemia (Ph+ ALL). After 4 years, 14 AP, 2 BP, and 1 ALL patients remained in the study, with a median duration of treatment of 10.2 months. Among AP patients, $57 \%$ achieved an overall 
Table 1 Best overall response observed for bosutinib-treated patients, for both first and subsequent treatment

\begin{tabular}{|c|c|c|c|c|}
\hline \multirow{2}{*}{$\begin{array}{l}\text { Response } \\
\text { (\% of evaluable patients) }\end{array}$} & \multicolumn{2}{|c|}{ Phase I/II trial $[26,27]$} & \multicolumn{2}{|l|}{ BELA trial $[29,30]$} \\
\hline & IMA-R $(n=200)$ & IMA-I $(n=88)$ & Bosutinib $(n=248)$ & Imatinib $(n=251)$ \\
\hline CHR 24 months & 85 & 82 & NR & NR \\
\hline CCyR 12 months & 36 & 50 & 70 & 68 \\
\hline 24 months & 46 & 54 & 87 & 81 \\
\hline MMR 12 months & 22 & 31 & 41 & 27 \\
\hline 24 months & 29 & 31 & 59 & 49 \\
\hline CMR 12 months & 16 & 13.5 & 12 & 3 \\
\hline 24 months & 25 & 32 & NR & NR \\
\hline PFS & 95 & 91 & NR & NR \\
\hline Progression to AP/BP & 5 & 1.1 & 2 & 5 \\
\hline OS (24 months) & 98 & 89 & 97 & 95 \\
\hline
\end{tabular}

$A P / B P$ accelerated/blastic phase, $B E L A$ bosutinib efficacy and safety in newly diagnosed CML, $C C y R$ complete cytogenetic response, $C H R$ complete hematological response, $C M R$ complete molecular response, IMA-I imatinib-intolerant, $I M A-R$ imatinib-resistant, $M M R$ major molecular response, $P F S$ progression-free survival, $N R$ not reported, $O S$ overall survival

hematologic response and 40\% a MCyR with a 4 -year probability of maintaining this response of $65 \%$; among BP patients, $28 \%$ achieved an hematologic response and 37\% a MCyR with a $21 \%$ probability of 4 -year duration. Responses were durable, suggesting possible use of this drug while awaiting transplant [29].

\section{SAFETY OF PHASE 1/2 STUDY}

The most common adverse side effects observed were gastrointestinal, for example diarrhea, nausea, vomiting, abdominal pain, rash, fever, fatigue and increased alanine aminotransferase. Most frequent grade 3/4 side effects were low incidence of diarrhea, increased ALT, and rash. Only $3 \%$ of patients experienced a pleural effusion related to the drug. Gastrointestinal side effects occurred early, and usually of low severity; transient diarrhea was managed with loperamide in $69 \%$ of cases, with temporarily interruptions in $15 \%$ of cases, or reduction of dose for $6 \%$ of patients. Forty-five percent of patients used antiemetic for nausea and 33\% for vomiting. Cardiac side effects were reported for $14 \%$ of patients, the most frequent being atrial fibrillation and palpitations; two patients discontinued as a result of cardiac side effects and one died of unrelated cardiac failure. With regard to hematologic toxicity, $24 \%$ of patients experienced grade $3 / 4$ thrombocytopenia in a median time of 21 days, whereas $17 \%$ experienced grade $3 / 4$ neutropenia and $8 \%$ anemia (Fig. 1). The most frequent laboratory abnormalities were elevated ALT (58\% overall and $10 \%$ as grade $3 / 4$ ), hypophosphatemia (43\% overall and $9 \%$ as grade $3 / 4)$, and elevated lipase ( $28 \%$ overall and $8 \%$ as grade $3 / 4)$. Also in the advanced phase of the disease, the most common side effects were gastrointestinal with diarrhea among $85 \%$ of $\mathrm{AP}$ patients and among $64 \%$ of BP patients, mostly of grade $1 / 2$. The most common serious adverse side effects reported were pneumonia 

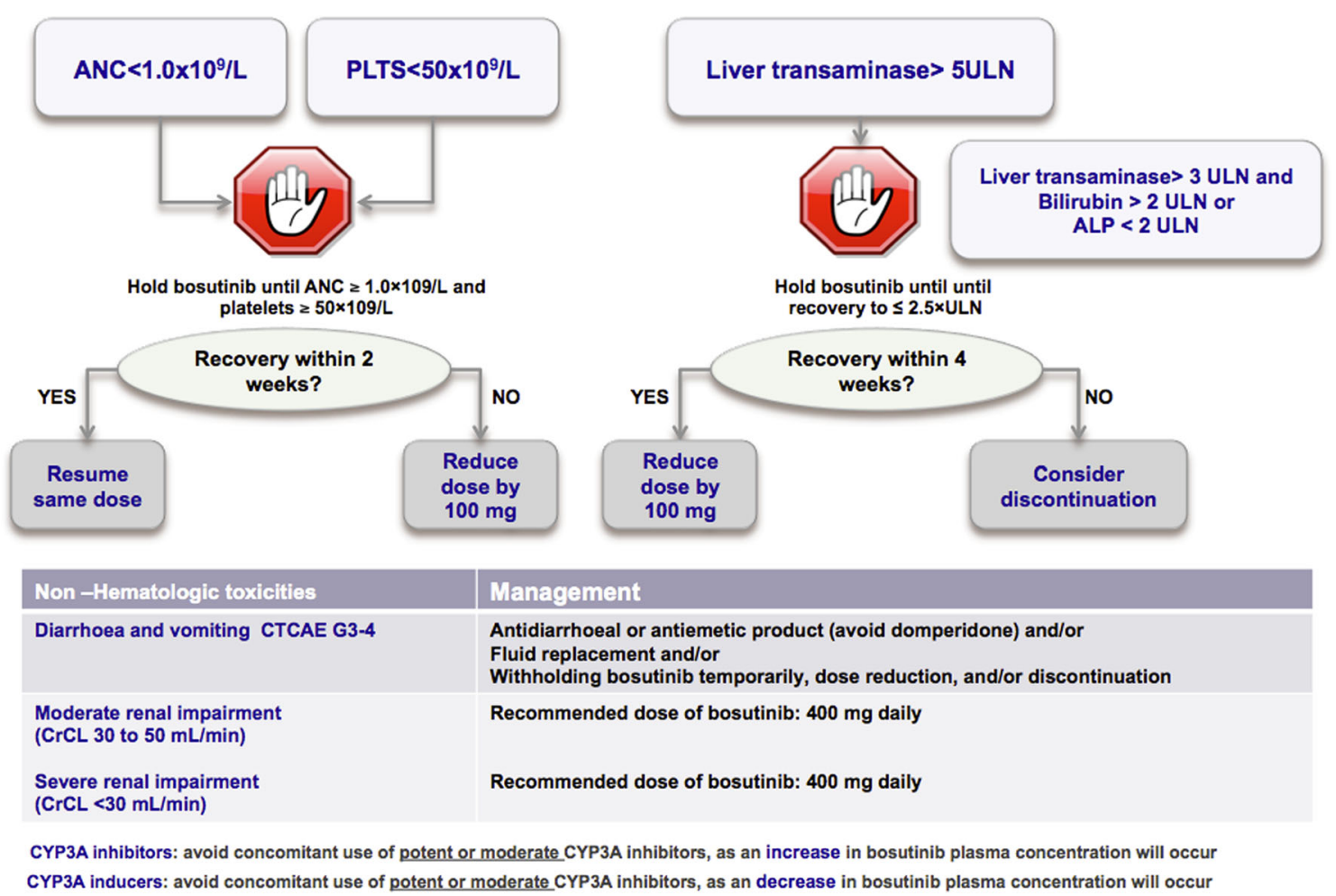

Fig. 1 Suggested management of the most frequent adverse side effects for patients treated with bosutinib

among AP patients ( 9 patients) and pyrexia for 6 BP patients [27] (Table 2).

\section{BOSUTINIB FOR NEWLY DIAGNOSED CML PATIENTS}

The BELA study was a phase 3 randomized trial that compared bosutinib with imatinib for newly diagnosed CP-CML patients [30]. Five hundred and two patients were randomly assigned 1:1 to bosutinib at a dose of $500 \mathrm{mg}$ per day or imatinib at $400 \mathrm{mg}$ per day. Follow-up at 1 year reported CCyR, the primary endpoint of the study, of $70 \%$ for bosutinib and $68 \%$ for imatinib, without significant difference. Median time to achieve CCyR was faster with bosutinib. MMR was higher for bosutinib (41\% compared with 27\% for imatinib) and CMR was also higher for bosutinib (12\% versus $3 \%)$. The median time to reach MMR was faster with bosutinib, 37 weeks compared with 72.3 weeks with imatinib. No differences were observed for different Sokal risk groups. Eleven side effects were recorded for bosutinib and 18 for imatinib, with estimated side-effect-free survival of $94 \%$ and $93 \%$, respectively. Two percent of patients with bosutinib experienced progression, compared with $10.4 \%$ in the imatinib group [30].

Recently, the trial was updated at 24 months: CCyR was 79\% with bosutinib and $80 \%$ with imatinib, whereas MMR was 59\% and 49\%, respectively. Responses were durable and since the previous report at 1 year no new cases of progression were detected with bosutinib whereas another four cases were observed with imatinib [31] (Table 1). 
Table 2 Adverse side effects associated with bosutinib treatment among imatinib-resistant, imatinib-intolerant, or previously untreated patients with chronic myeloid leukemia

\begin{tabular}{|c|c|c|c|c|}
\hline \multirow{2}{*}{$\begin{array}{l}\text { Adverse side effects \% } \\
\text { All (\% G3-4) }\end{array}$} & \multicolumn{2}{|c|}{ Phase I/II trial [27] } & \multicolumn{2}{|l|}{ BELA trial [30] } \\
\hline & IMA-I $(n=88)$ & IMA-R $(n=200)$ & Bosutinib $(n=248)$ & Imatinib $(n=251)$ \\
\hline Thrombocytopenia & $66(21)$ & $70(32)$ & $28(13)$ & $28(14)$ \\
\hline Anemia & $91(12)$ & $86(18)$ & $25(8)$ & $22(6)$ \\
\hline Neutropenia & $49(14)$ & $51(24)$ & $13(8)$ & $29(16)$ \\
\hline Diarrhea & $84(9)$ & $85(13)$ & $70(12)$ & $25(1)$ \\
\hline Nausea & $42(0)$ & $51(5)$ & $32(1)$ & $36(0)$ \\
\hline Vomiting & $35(2)$ & $41(9)$ & $32(3)$ & $16(0)$ \\
\hline Rash & $32(9)$ & $41(11)$ & $24(2)$ & $19(1)$ \\
\hline Pyrexia & $27(1)$ & $16(0)$ & $18(1)$ & $12(1)$ \\
\hline Abdominal pain & $23(1)$ & $25(2)$ & $13(1)$ & $7(<1)$ \\
\hline Fatigue & $23(1)$ & $25(2)$ & $13(1)$ & $14(1)$ \\
\hline Elevated AST & $49(4)$ & $55(7)$ & $27(8)$ & $9(3)$ \\
\hline Elevated ALT & $55(10)$ & $66(11)$ & $32(18)$ & $8(3)$ \\
\hline Elevated creatinine & $37(1)$ & $41(0)$ & NR & NR OK \\
\hline
\end{tabular}

$A S T$ aspartate aminotransferase, $A L T$ alanine aminotransferase, $B E L A$ bosutinib efficacy and safety in newly diagnosed CML, IMA-I imatinib-intolerant, IMA-R imatinib-resistant, $N R$ not reported

\section{SAFETY OF BOSUTINIB AMONG NEWLY DIAGNOSED CP PATIENTS}

A recently published update of the BELA trial reported safety analysis after more than 30 months of follow-up. In the bosutinib group gastrointestinal side effects were more frequent, for example diarrhea $70 \%$ compared with $26 \%$ in the imatinib arm and vomiting in $33 \%$ versus $16 \%$, respectively. As in the phase $1 / 2$ trial, elevation of alanine aminotransferase and aspartate aminotransferase was observed in the bosutinib group (33\% and $28 \%$ versus $9 \%$ and $10 \%$, respectively). Less common with bosutinib were recorded edema (7\% versus 26\%), musculoskeletal pain (cramp 5\% versus $22 \%$, bone pain $4 \%$ versus $11 \%$ ), and neutropenia (13\% v3ersus 30\%). No significant difference was noted between the two groups.
Gastrointestinal events were usually transient, manageable with concomitant medications, and usually occurred during the first months of treatment. In particular, diarrhea was managed by dose modification and/or concomitant medication [33] (Table 2).

\section{CARDIOVASCULAR SAFETY ANALYSIS}

Retrospective analysis evaluating cardiac toxicity data from the 2 studies (BELA and study 200) has been reported. Patients were excluded at study entry if they required medications that prolong the QT interval, had a history of significant/uncontrolled cardiac disease (congestive heart failure, uncontrolled angina, or hypertension within 3 months, myocardial infarction within 12 months, 
clinically significant ventricular arrhythmia, diagnosis or suspected congenital or acquired prolonged QT syndrome, history of prolonged QTc, or unexplained syncope), or had average QTc $>0.45 \mathrm{~s}$ at screening or uncorrected hypomagnesemia or hypokalemia. Treatment-emergent adverse side effects were monitored throughout the studies and coded according to NCI CTCAE version 3.0.

Incidence of exposure-adjusted cardiac adverse side effects was 0.059 for bosutinib and 0.042 for imatinib in the phase 3 BELA study and 0.096 for bosutinib in the phase $1 / 2$ study. The most common cardiac side effect experienced by bosutinib-treated patients in both studies was cardiac arrhythmias (5.7\% overall; grade $3 / 4,1.5 \%)$. Cardiac arrhythmias occurred for $4.0 \%$ and $2.0 \%$ (grade $3 / 4,0.4 \%$ and $0 \%$ of bosutinib-treated and imatinib-treated patients, respectively, in the phase 3 BELA study, and in $6.5 \%$ (grade $3 / 4$, $1.9 \%$ ) of bosutinib-treated patients in the phase $1 / 2$ study.

Incidence of heart failures was uncommon overall $(2.9 \%$ of all bosutinib-treated patients; grade $3 / 4,1.7 \%$; it was similar for bosutinib $(0.8 \%$; grade $3 / 4,0.8 \%)$ and imatinib $(0.8 \%$; grade $3 / 4,0 \%$ ) in the phase 3 BELA study. The incidence of newly emergent side effects decreased with longer duration of treatment.

In the phase 3 BELA study, discontinuations because of cardiac side effects were numerically higher for bosutinib-treated than for imatinib-treated patients $(1.6 \%$ versus $0 \%$, respectively). Reasons for discontinuation for the 4 bosutinib-treated BELA patients were pericardial effusion, right bundle branch block, congestive cardiac failure, and ECG QT prolonged.

In both bosutinib studies, few patients (2.0\%; grade $3 / 4,0.9 \%$ ) discontinued bosutinib because of cardiac side effects.
In the phase 3 BELA study cardiac side effects leading to dose delays were not significantly more frequent among bosutinib-treated patients than among imatinib-treated patients $(3.6 \%$ versus $1.6 \%$, respectively; grade $3 / 4,0.8 \%$ versus $0 \%$ ). Of 32 patients from both studies whose bosutinib dose was delayed because of adverse side effects, 26 were re-challenged and 6 discontinued bosutinib permanently because of a cardiac side effect.

Analysis of predisposing factors to cardiac events showed that age $>65$ years, previous history of cardiac disorders, ECOG $>0$ in the phase $1 / 2$ study and history of hypertension in phase 3 trial, or hypercholesterolemia (in both studies) were significantly associated. Indeed, the incidence of vascular emergent side effects with bosutinib was comparable with that with imatinib in the BELA study [33]. With the exception of hypertension, which was common with bosutinib (in a combined analysis any grade $<7 \%$; grade $3 / 4<2 \%$ ), the incidence of vascular side effects was particularly low [34].

\section{CROSS-INTOLERANCE BETWEEN BOSUTINIB AND PREVIOUS TKI}

Analysis was conducted to investigate the potential cross-intolerance of bosutinib with previous TKI when used as second or third line treatment. Of 143 patients previously treated with imatinib, 22 (20 in CP and 2 in advanced phase) discontinued the drug for the same effect (prevalently cytopenias). Seventy-one patients previously treated with dasatinib received bosutinib for intolerance: 7 patients in $\mathrm{CP}$ and 1 in $\mathrm{AP}$ experienced the same side effect (thrombocytopenia and pleural effusions). 
Of 7 patients intolerant of nilotinib, 3 patients then discontinued bosutinib for thrombocytopenia also experienced as a result of the previous treatment. This data showed the absence of cross-intolerance between bosutinib and the other drugs [35].

\section{WHICH IS THE IDEAL PLACE FOR BOSUTINIB IN THE CONTEST OF SEVERAL CHOICES?}

Considering that agreement on the application of each TKI for management of CML is far from being established, personal perspectives rather specific recommendations for bosutinib use can be drawn.

Undoubtedly, one advantage of this drug is its favorable safety profile. A low incidence of some adverse side effects common with other TKI makes bosutinib a good choice for patients intolerant to other TKI with comorbidities or cardiovascular risk factors. Moreover, bosutinib is active against many BCR-ABL kinase domain mutations resistant to imatinib, dasatinib, and nilotinib, with the exception of T315I and V299L.

Patients who experience treatment failure with a second-generation TKI (2G-TKI) as first or second-line treatment seem to gain limited benefit from sequential use of 2G-TKI; Lipton and colleagues recently reviewed published data and concluded that the probability of complete cytogenetic response ranged from $22 \%$ to $26 \%$ for the 2G-TKI examined [36].

In conclusion, with the increasing therapeutic options available for CML and continually increasing treatment objectives, healthcare providers have the opportunity to design patient-tailored strategies in accordance with individual characteristics such as age, comorbidities, life-style, treatment history, disease stage, and TKI toxicity profile.

\section{ACKNOWLEDGMENTS}

No funding or sponsorship was received for this study or publication of this article. Both authors had full access to all of the data in this study and take complete responsibility for the integrity of the data and the accuracy of the data analysis. Both named authors meet the International Committee of Medical Journal Editors (ICMJE) criteria for authorship of this manuscript, take responsibility for the integrity of the work as a whole, and have given final approval for the version to be published.

Conflict of interest. Massimo Breccia and Gianni Binotto declare no conflict of interest.

Compliance with ethics guidelines. This article is based on previously conducted studies and does not involve any new studies of human or animal subjects performed by either of the authors.

Open Access. This article is distributed under the terms of the Creative Commons Attribution-NonCommercial 4.0 International License (http://creativecommons.org/licenses/ by-nc/4.0/), which permits any noncommercial use, distribution, and reproduction in any medium, provided you give appropriate credit to the original author(s) and the source, provide a link to the Creative Commons license, and indicate if changes were made.

\section{REFERENCES}

1. Kim LC, Song L, Haura EB. Src kinases as therapeutic targets for cancer. Nat Rev Clin Oncol. 2009;6:587?95.

2. Guarino M. Src signaling in cancer invasion. J Cell Physiol. 2010;223:14?26. 
3. Druker BJ, Tamura S, Buchdunger E, et al. Effects of a selective inhibitor of the abl tyrosine kinase on the growth of Bcr-Abl positive cells. Nat Med. 1996;2:561?6.

4. Deininger MW, Goldman JM, Melo JV. The molecular biology of chronic myeloid leukemia. Blood. 2000;96:3343?56.

5. Druker BJ, Talpaz M, Resta DJ, et al. Efficacy and safety of a specific inhibitor of the Bcr-Abl tyrosine kinase in chronic myeloid leukemia. N Engl J Med. 2001;344:1031?7.

6. Hughes T, White D. Which tki? An embarrassment of riches for chronic myeloid leukemia patients. Hematol Am Soc Hematol Educ Progr. 2013;1:168?75.

7. Hochhaus A, O?Brien SG, Guilhot F, et al. Six-year follow-up of patients receiving imatinib for the first-line treatment of chronic myeloid leukemia. Leukemia. 2009;23:1054?61.

8. Castagnetti F, Gugliotta G, Breccia $M$, et al. Long-term outcome of chronic myeloid leukemia patients treated frontline with imatinib. Leukemia. 2015. doi:10.1038/leu.2015.152 (Epub ahead of print).

9. Kantarjian HM, Giles FJ, Bhalla KN, et al. Nilotinib is effective in patients with chronic myeloid leukemia in chronic phase after imatinib resistance or intolerance: 24-month follow-up results. Blood. 2011;117:1141?5.

10. Shah NP, Guilhot F, Cortes JE, et al. Long-term outcome with dasatinib after imatinib failure in chronic-phase chronic myeloid leukemia: follow-up of a phase 3 study. Blood. 2014;123:2317?24.

11. Larson RA, Hochhaus A, Hughes TP, et al. Nilotinib versus imatinib in patients with newly diagnosed Philadelphia Chromosome-Positive chronic myeloid leukemia in chronic phase: enestnd 3-year follow-up. Leukemia. 2012;26:2197?203.

12. Kantarjian HM, Shah NP, Cortes JE, et al. Dasatinib or imatinib in newly diagnosed chronic-phase chronic myeloid leukemia: 2-year follow-up from a randomized phase 3 trial (dasision). Blood. 2012;119:1123?9.

13. Boschelli DH, Ye F, Wang YD, et al. Optimization of 4-phenylamino-3-quinolinecarbonitriles as potent inhibitors of Src kinase activity. J Med Chem. 2001;44:3965?77.

14. Golas JM, Arndt K, Etienne C, et al. Ski-606, a 4-anilino-3-quinolinecarbonitrile dual inhibitor of Src and Abl kinases, is a potent antiproliferative agent against chronic myelogenous leukemia cells in culture and causes regression of K562 xenografts in nude mice. Cancer Res. 2003;63:375?81.

15. Puttini M, Coluccia AM, Boschelli F, et al. In vitro and in vivo activity of SKI-606, a novel Src-Abl inhibitor, against imatinib-resistant $\mathrm{Bcr}-\mathrm{Abl}+$ neoplastic cells. Cancer Res. 2006;66:11314?22.

16. Konig H, Holyoake TL, Bhatia R. Effective and selective inhibition of chronic myeloid leukemia primitive hematopoietic progenitors by the dual Src/Abl kinase inhibitor SKI-606. Blood. 2008;111:2329?38.

17. Remsing Rix LL, Rix U, Colinge J, et al. Global target profile of the kinase inhibitor bosutinib in primary chronic myeloid leukemia cells. Leukemia. 2009;23:477?85.

18. Hsyu PH, Mould DR, Abbas R, et al. Population pharmacokinetic and pharmacodynamic analysis of bosutinib. Drug Metab Pharmacokinet. 2014;29:441?8.

19. Abbas R, Hug BA, Leister C, et al. A phase I ascending single-dose study of the safety, tolerability, and pharmacokinetics of bosutinib (SKI-606) in healthy adult subjects. Cancer Chemother Pharmacol. 2012;69:221?7.

20. Shen AQ, Wilson NM, Gleason SL, et al. Bosutinib in the treatment of patients with philadelphia chromosome-positive $(\mathrm{Ph}+)$ chronic myelogenous leukemia: an overview. Ther Adv Hematol. 2014;5:13?7.

21. Abbas R, Hug BA, Leister C, et al. Effect of ketoconazole on the pharmacokinetics of oral bosutinib in healthy subjects. J Clin Pharmacol. 2011;51:1721?7.

22. Abbas R, Boni J, Sonnichsen D. Effect of rifampin on the pharmacokinetics of bosutinib, a dual SRC/ ABL tyrosine kinase inhibitor, when administered concomitantly to healthy subjects. Drug Metabol Personal Ther. 2015;30:57?63.

23. Abbas R, Leister C, Sonnichsen D. A clinical study to examine the potential effect of lansoprazole on the pharmacokinetics of bosutinib when administered concomitantly to healthy subjects. Clin Drug Investig. 2013;33:589?95.

24. Hsyu PH, Mould DR, Upton RN, et al. Pharmacokinetic-pharmacodynamic relationship of bosutinib in patients with chronic phase chronic myeloid leukemia. Cancer Chemother Pharmacol. 2013;71:209?18.

25. Abbas R, Chalon S, Leister C, et al. Evaluation of the pharmacokinetics and safety of bosutinib in 
patients with chronic hepatic impairment and matched healthy subjects. Cancer Chemother Pharmacol. 2013;71:123?32.

26. BOSULIF $^{\circledR} \quad$ (bosutinib). Full prescribing information. New York: Pfizer Labs; 2013. (www. bosulif.com).

27. Cortes JE, Kantarjian HM, Brummendorf TH, et al. Safety and efficacy of bosutinib (SKI-606) in chronic phase philadelphia chromosome-positive chronic myeloid leukemia patients with resistance or intolerance to imatinib. Blood. 2011;118:4567?76.

28. Gambacorti-Passerini C, Brummendorf TH, Kim DW, et al. Bosutinib efficacy and safety in chronic phase chronic myeloid leukemia after imatinib resistance or intolerance: minimum 24-month follow-up. Am J Hematol. 2014;89:732?42.

29. Gambacorti-Passerini C, Kantarjian HM, Kim DW, et al. Long-term efficacy and safety of bosutinib in patients with advanced leukemia following resistance/intolerance to imatinib and other tyrosine kinase inhibitors. Am J Hematol. 2015 (Epub ahead of print).

30. Cortes JE, Kim DW, Kantarjian HM, et al. Bosutinib versus imatinib in newly diagnosed chronic-phase chronic myeloid leukemia: results from the BELA trial. J Clin Oncol. 2012;30:3486?92.

31. Brummendorf TH, Cortes JE, de Souza CA, et al. Bosutinib versus imatinib in newly diagnosed chronic-phase chronic myeloid leukaemia: results from the 24-month follow-up of the BELA trial. Br J Haematol. 2015;168:69?81.

32. Gambacorti-Passerini C, Cortes JE, Lipton JH, et al. Safety of bosutinib versus imatinib in the phase 3 BELA trial in newly diagnosed chronic phase chronic myeloid leukemia. Am J Hematol. 2014;89:947?53.

33. Gambacorti-Passerini C, Kantarjian HM, Khoury HJ, et al. Long-term assessment of cardiac toxicity in patients With $\mathrm{Ph}+$ leukemias treated with bosutinib. EHA. 2014, poster\#903.

34. Cortes JE, Kantarjian HM, Khoury HJ, et al. Long-term evaluation of vascular toxicity in patients with $\mathrm{Ph}+$ leukemias treated with bosutinib. EHA. 2014, poster\#900.

35. Cortes JE, Lipton JH, Kantarjian HM, et al. Evaluation of cross-intolerance between bosutinib and prior tyrosine kinase inhibitor therapy in patients with philadelphia chromosome?positive $(\mathrm{Ph}+)$ leukemia. EHA. 2013, poster\#151.

36. Lipton JH, Shah D, Tongbram V, et al. Comparative efficacy among 3rd line post-imatinib chronic phase-chronicmyeloid leukemia (CP-CML) patients after failure of dasatinib or nilotinib tyrosine kinase inhibitors. ASH. 2014, Abs \# 4551. 